\title{
Transitions to Electrochemical Turbulence
}

\author{
Hamilton Varela, ${ }^{1}$ Carsten Beta, ${ }^{1}$ Antoine Bonnefont, ${ }^{1,2}$ and Katharina Krischer ${ }^{1,2, *}$ \\ ${ }^{1}$ Fritz-Haber-Institut der MPG, Faradayweg 4-6, D-14195 Berlin, Germany \\ ${ }^{2}$ Technische Universität München, Physik Department E19, James Franck Strasse 1, D-85748 Garching, Germany
}

(Received 14 June 2004; published 4 May 2005)

\begin{abstract}
We report experimental evidence of transitions from limit cycle oscillations through a phase turbulent regime to space-time defect turbulence in a spatially (quasi-)one-dimensional electrochemical system with nonlocal coupling. The transitions are characterized in terms of the defect density, the KarhunenLoève decomposition dimension, and a measure of the degree of spatial correlation in the data. Furthermore, these quantities give the first experimental confirmation that the spatial coupling range in electrochemical systems indeed depends on the distance between the working and the counterelectrode.
\end{abstract}

PACS numbers: 82.40.Bj, 05.45.-a, 68.08.-p

Systems that are driven far from thermodynamic equilibrium may exhibit spatiotemporally chaotic states, characterized by a finite correlation length in both space and time [1]. For the last decade, it has been a challenge to reveal mechanisms that govern transitions to spatiotemporal chaos and to establish methods for characterizing such high-dimensional disordered states. Much of this work is theoretical, calling now for systematic experimental studies.

Previous theoretical investigations mostly focused on the complex Ginzburg-Landau equation (CGLE), a generic model for diffusively coupled oscillators close to a supercritical Hopf bifurcation. In the framework of the CGLE, a well known source of spatiotemporal chaos (widely referred to as chemical turbulence in this context) is the Benjamin-Feir (BF) instability, see, e.g., [1-3]. When a one-dimensional system obeying the CGLE is driven beyond the BF instability, it first develops phase turbulence, where the local oscillation phase shows weak irregular fluctuations. Farther away from the instability, defect turbulence occurs, which is characterized by the presence of phase dislocations [4].

Experimental studies of spatiotemporal chaos are still rare. They mostly focus on systems and situations for which the CGLE proved to be a good model, such as some hydrodynamic systems (see, e.g., [5]), or chemical systems [6-12]. All of these experimental examples involve spatially two-dimensional systems and identify either routes to spatiotemporal chaos different from the above-mentioned scenario or aim at suppressing turbulence, rather than at the study of its emergence. In this Letter, we report experimental observations of transitions from relaxation oscillations to defect turbulence in a (quasi-)one-dimensional electrochemical system. Spatial coupling in electrochemical systems is nonlocal and, thus, bridges the gap between local nearest neighbor coupling and global "all-to-all" coupling. The observed spatiotemporal chaos is migration induced, i.e., induced by nonlocal coupling. To stress the similarity of these chaotic states with chemical turbulence observed in the BF- unstable region of reaction-diffusion systems, they will be referred to as electrochemical turbulence. Finally, by characterizing different turbulent states we confirm a theoretical prediction saying that the range of the spatial coupling in an electrochemical system depends on the distance between the working (WE) and the counter (CE) electrode.

Oscillatory electrochemical systems can be considered as active distributed media and are mathematically described by a set of coupled partial differential equations. They differ from reaction-diffusion systems only in the spatial coupling term of the electric potential drop across the electrode/electrolyte interface, $\phi_{\mathrm{DL}}$, the main variable in electrochemical systems [13]. In many of these systems, and also the one considered here, $\phi_{\mathrm{DL}}$ is an autocatalytic variable and its evolution equation in dimensionless form reads

$$
\frac{\partial \phi_{\mathrm{DL}}(x)}{\partial t}=f\left(\phi_{\mathrm{DL}}, c_{i}\right)-\left.\sigma\left(\frac{1}{\beta} \frac{\partial \phi}{\partial z}-\frac{\phi}{\beta}\right)\right|_{z=\mathrm{WE}}
$$

where $c_{i}$ are concentrations of species reacting at the electrode, their diffusion parallel to the electrode being in general negligible.

The last term of Eq. (1) describes the nonlocal migration coupling, which is mediated through the electric potential in the electrolyte, $\phi(x, z)$, obtained by solving Laplace's equation $[14,15]$ ( $x$ and $z$ being the coordinates parallel and perpendicular to the electrode, respectively, and $z=\mathrm{WE}$ a position at the WE; $\sigma$ is the dimensionless conductivity). Moreover, Eq. (1) predicts a peculiar property of spatial coupling in electrochemical systems, namely, that its range, i.e., the distance over which a perturbation at a given point is felt instantaneously and with a finite strength, increases with the distance between the WE and the CE [14]. This is reflected in the dependence of Eq. (1) on the parameter $\beta$. For a ring-shaped working electrode, as used in the present study, $\beta$ is the ratio between the circumference of the electrode and the distance between the WE and the CE. The coupling can be expressed in terms of the integral over a coupling function, $H\left(\left|x-x^{\prime}\right|\right)$, as first discussed in [16], 


$$
\begin{aligned}
-\left.\left(\frac{1}{\beta} \frac{\partial \phi}{\partial z}-\frac{\phi}{\beta}\right)\right|_{z=\mathrm{WE}} & \int_{\mathrm{WE}} H\left(\left|x-x^{\prime}\right|\right)\left[\phi_{\mathrm{DL}}\left(x^{\prime}\right)\right. \\
& \left.-\phi_{\mathrm{DL}}(x)\right] d x^{\prime} .
\end{aligned}
$$

In Fig. 1(a), $H\left(\left|x-x^{\prime}\right|\right)$ is shown for two different coupling ranges.

The system investigated is the electrooxidation of hydrogen on $\mathrm{Pt}$ in the presence of $\mathrm{Cu}^{2+}$ and $\mathrm{Cl}^{-}$ions [17]. The WE consisted of a polycrystalline Pt ring. The voltage between the WE (which was grounded) and a potential microprobe (PP) which was located immediately beneath the ring [see Fig. 1(b)] was measured, the obtained signal being a very good approximation of $\phi_{\mathrm{DL}}(x)$. Rotating the ring over the microprobe, the temporal evolution of spatial profiles of $\phi_{\mathrm{DL}}(x, t)$ along the angular direction $x$ are obtained. The rotation of the ring ensured also a defined mass transport of $\mathrm{H}_{2}$, as well as $\mathrm{Cu}^{2+}$ and $\mathrm{Cl}^{-}$ions from the bulk electrolyte to the reaction plane at the WE. Note, however, that the rotation did not interfere with the pattern formation, which occurs at the solid-liquid interface, i.e., within a boundary layer that is firmly attached to and rotates with the WE, as also confirmed in independent experiments [18]. The RE was placed in a separate compartment whose junction to the main cell was below the CE. Experiments were conducted for two different distances between the WE and the CE, namely, 40 (case 1) and $5 \mathrm{~mm}$ (case 2) to realize different coupling ranges (Fig. 1).

For the chosen composition of the electrolyte and a constant applied voltage $U$ between the WE and the RE the system exhibited nonstationary states over a large voltage range for both large (case 1) and small (case 2) separations of WE and CE. In both cases, the dynamics was more regular at low values of $U$, i.e., low driving force for
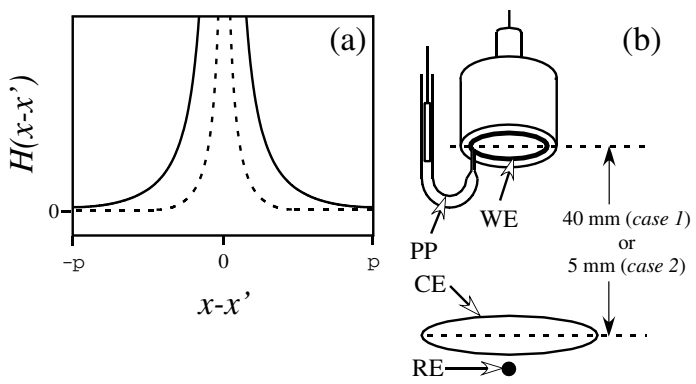

FIG. 1. (a) Coupling function, $H\left(\left|x-x^{\prime}\right|\right)$, plotted versus the distance $\left(x-x^{\prime}\right)$ from a reference point $x$ for a 1-dimensional ring electrode and two different coupling ranges; solid curve: nonlocal coupling, dashed curve: more localized coupling. (b) Experimental setup; WE: working electrode (mean circumference: $85 \mathrm{~mm}$; width: $1 \mathrm{~mm}$ ), CE: counterelectrode, RE: reference electrode ( $\mathrm{Hg} / \mathrm{Hg}_{2} \mathrm{SO}_{4}$, sat.), located below the plane of the $\mathrm{CE}$ in both cases, PP: potential probe. The WE was rotated at $20 \mathrm{~Hz}$ and the spatiotemporal evolution of the interfacial potential was obtained by the PP with a resolution of 50 points per rotation. See Ref. [23] for further experimental details. the oxidation of hydrogen, and became increasingly complex in space and time for increasing $U$. Parts of experimental current time series and spatiotemporal plots of the interfacial potential are depicted in Fig. 2 for the two cases and three different values of $U$. In case 1 , the current time series is periodic at low values of $U$, the interfacial potential being almost completely synchronized along the entire electrode (a). With increasing $U$, the initially small and regular spatial modulations become more pronounced and irregular (b) until eventually oscillations break up into domains with decreasing average length scale. A typical state in the strongly irregular regime is depicted in (c).

Also for a closer distance between the WE and the CE (case 2), the dynamics becomes more irregular with increasing $U$. However, in this case, even at the lowest values of $U$, oscillations are not strictly periodic and spatial modulations are already pronounced, Fig. 2(d). The transition to developed turbulence occurs in a smaller interval of $U$, and the minimal length scale is smaller than in case 1 .

To further substantiate the nature of the transitions, we transformed the potential values $\phi_{\mathrm{DL}}(x, t)$ into amplitude and phase variables following a variant of the analytic signal approach [19-21]. First, the analytic signal

$$
\xi(\boldsymbol{x}, t)=\phi_{\mathrm{DL}}(\boldsymbol{x}, t)+i \tilde{\phi}_{\mathrm{DL}}(\boldsymbol{x}, t)
$$

is computed, where $\tilde{\phi}_{\mathrm{DL}}(\boldsymbol{x}, t)$ is the Hilbert transform of $\phi_{\mathrm{DL}}(\boldsymbol{x}, t)$

$$
\tilde{\phi}_{\mathrm{DL}}(\boldsymbol{x}, t)=\pi^{-1} \int_{-\infty}^{+\infty}\left(t-t^{\prime}\right)^{-1} \phi_{\mathrm{DL}}\left(\boldsymbol{x}, t^{\prime}\right) d t^{\prime} .
$$

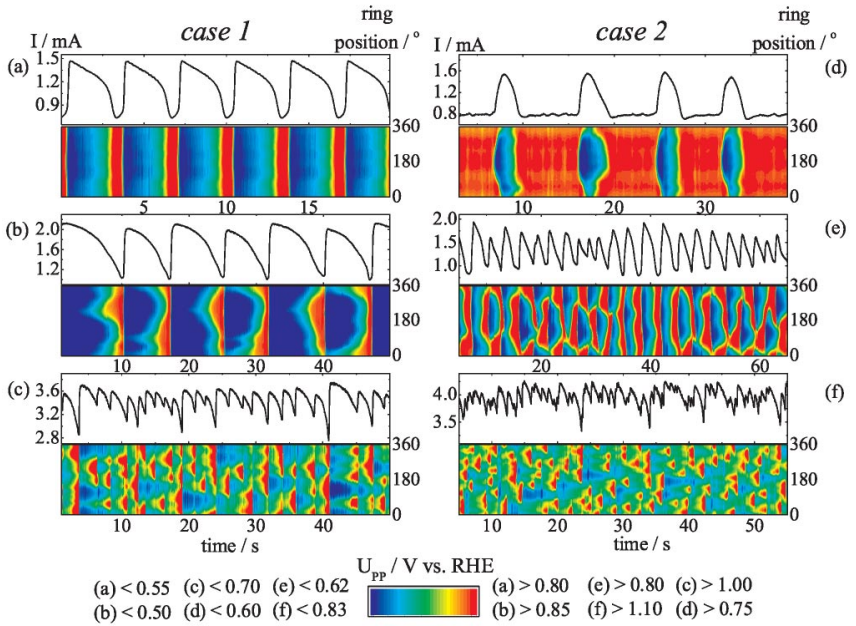

FIG. 2 (color online). Global current time series and spatiotemporal evolution of the interfacial potential, $\phi_{\mathrm{DL}}$, as a function of position on the ring and time for different values of the applied voltage $U$ and electrode placement corresponding to case 1 [plates (a), (b), and (c)] and case 2 [(d), (e), and (f)]. $U$ : (a) $1.06 \mathrm{~V}$, (b) $1.19 \mathrm{~V}$, (c) $2.14 \mathrm{~V}$, (d) $0.82 \mathrm{~V}$, (e) $0.89 \mathrm{~V}$, and (f) $1.54 \mathrm{~V}$. Electrolyte: $\mathrm{H}_{2}$-saturated, aqueous $0.5 \mathrm{mM} \mathrm{H}_{2} \mathrm{SO}_{4}$ solution containing $0.1 \mathrm{mM} \mathrm{HCl}$ and $0.01 \mathrm{mM} \mathrm{CuSO}_{4}$. A continuous flow of $\mathrm{H}_{2}$ was maintained throughout the experiments. 
The phase $\varphi$ is then directly calculated from $\xi, \varphi=\arg \xi$, whereas the amplitude variable $A=\rho / \rho_{\text {ref }}$ is obtained from the modulus of $\xi$ through normalization by a reference amplitude $\rho_{\text {ref }}(\varphi)$. The latter was extracted from the analytical signal by averaging $\rho=|\xi|$ separately inside each of 100 equidistant intervals of $\varphi$.

An example of phase and amplitude representations of a strongly irregular data set belonging to case 2 is shown in Figs. 3(a) and 3(b). Clearly, there are locations at which the amplitude vanishes and the phase changes abruptly, see circles in Figs. 3(a) and 3(b). This indicates the presence of space-time defects [4]. From the phase representations we determined the density of such defects for the two transitions. The results are summarized in Fig. 3(c) where the defect density is plotted versus the applied voltage for case 1 (solid circles) and case 2 (open circles). In both data sets, the defect density increases with increasing voltage starting from zero, revealing that both series exhibit a transition into a defect turbulent regime. Besides, in both cases a regime dominated by irregular phase modulations in the absence of defects is found at lower voltages, see Figs. 2(b) and 2(d). In case 1, moreover, we observe periodic limit cycle oscillations at the lowest value of $U \mathrm{cf}$. Fig. 2(a), preceding the irregular phase modulated regime. Hence, the two series are indeed experimental manifestations of a transition from phase to defect turbulence, in case 1 even a transition from a periodic limit cycle to defect turbulence via a phase turbulent regime is captured.
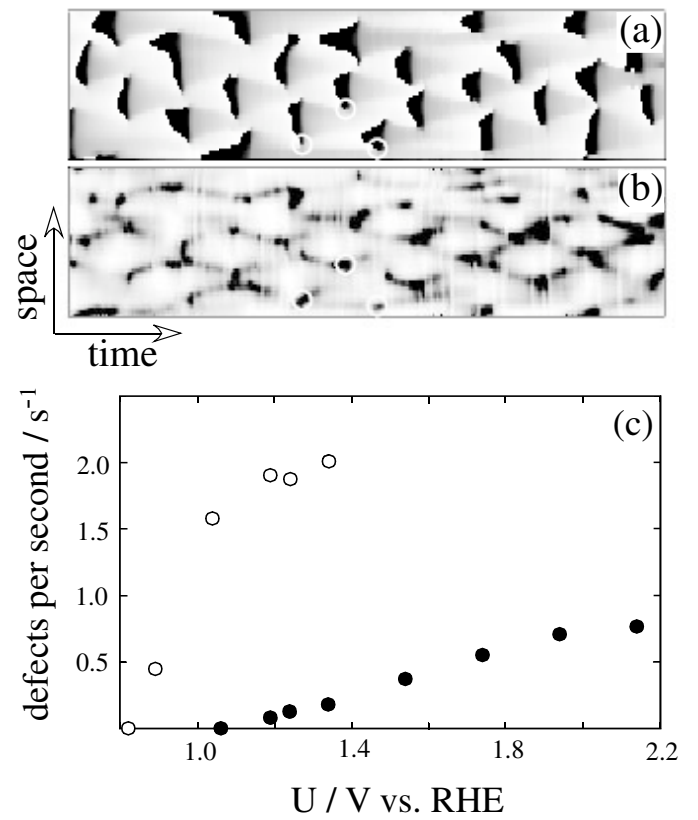

FIG. 3. (a) Phase and (b) amplitude representations of spatiotemporal data obtained for case 2 at $U=1.04 \mathrm{~V}$. Circles in (a) and (b) exemplify the locations of phase defects. The displayed time interval is $22.5 \mathrm{~s}$. (c) Defect density versus the applied voltage for case 1 (solid circles) and case 2 (open circles).
Figure 3(c) also yields information about the difference of the two cases: Not only does the defect density increase much faster in case 2 than in case 1, but series 2 also reaches a final defect density that is about two and a half times larger than in series 1 . Note that both series were recorded up to values of $U$ at which the Pt surface started to oxidize which determines the upper meaningful value of the voltage. Thus, in case 2 the system becomes more turbulent than in case 1, indicating that the dimension of the attractor is larger in case 2.

The Karhunen-Loève decomposition (KLD) dimension, $D_{\mathrm{KLD}}$, represents a measure of the complexity of spatiotemporal data and was shown to scale with the volume size for extensively chaotic states [22]. Our space-time data $\phi_{\mathrm{DL}}\left(x_{i}, t_{k}\right)$ are known at $M$ uniformly distributed instants in time $(M \geq 6000)$ and $N$ discrete equidistant points in space $(N=50)$. Let $\phi_{\mathrm{DL}}^{\prime}\left(x_{i}, t_{k}\right)$ denote the deviations of $\phi_{\mathrm{DL}}\left(x_{i}, t_{k}\right)$ from their time average $\left\langle\phi_{\mathrm{DL}}\left(x_{i}, t_{k}\right)\right\rangle$ (averaged over index $k)$ : $\phi_{\mathrm{DL}}^{\prime}\left(x_{i}, t_{k}\right)=\phi_{\mathrm{DL}}\left(x_{i}, t_{k}\right)-\left\langle\phi_{\mathrm{DL}}\left(x_{i}, t_{k}\right)\right\rangle$. Then, the elements $r_{i j}$ of the two-point spatial correlation matrix $\boldsymbol{R}$ are defined as $r_{i j}=\left\langle\phi_{\mathrm{DL}}^{\prime}\left(x_{i}\right) \phi_{D L}^{\prime}\left(x_{j}\right)\right\rangle$, where the cornered brackets again denote the time average. The dimension $D_{\mathrm{KLD}}$ measures the number of linear eigenmodes of $\boldsymbol{R}$ needed to capture a certain percentage $P$ of the statistical variance of the data. Qualitatively, an increase in the number of degrees of freedom of an attractor will be reflected by an increase in $D_{\mathrm{KLD}}$ [22].

In Fig. 4, the values of $D_{\mathrm{KLD}}$ are shown for the two series of experiments and for a fixed percentage of $P=95 \%$. As for the defect density, there is a clear-cut difference between the two sequences. In case 2 (open circles), $D_{\text {KLD }}$ increases more rapidly and reaches a plateau value, which lies well above the highest $D_{\mathrm{KLD}}$ determined for case 1 (solid circles). The larger value of $D_{\mathrm{KLD}}$ evidences the more turbulent character of the dynamics in case 2 as well as a smaller length scale of dissipation below which excitations are damped. Comparing the changes in $D_{\mathrm{KLD}}$ and in the defect density with increasing $U$, the development of the two quantities is very similar in case 2 . For

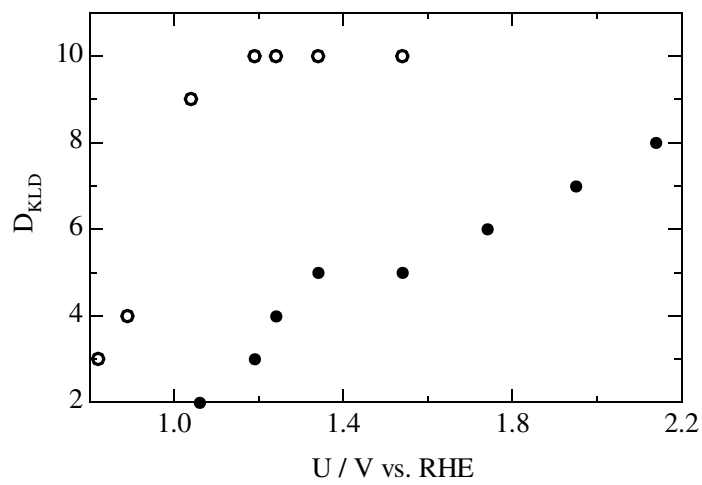

FIG. 4. Karhunen-Loève decomposition dimension, $D_{\mathrm{KLD}}$, as a function of the applied voltage for case 1 (solid circles) and case 2 (open circles). 


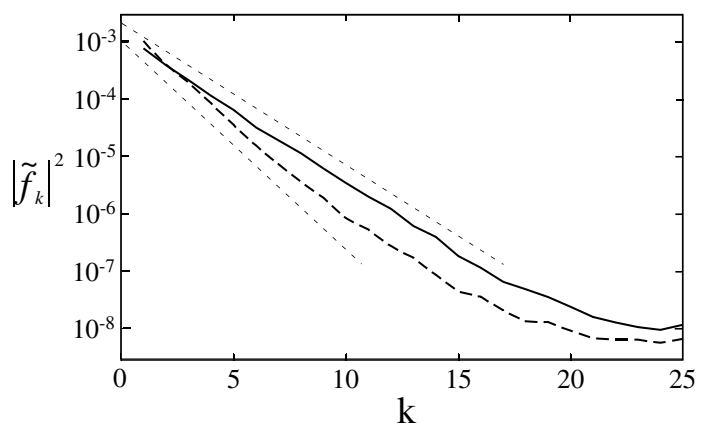

FIG. 5. Time averaged spatial power spectra in the fully developed defect turbulent regime for a data set of both the nonlocally coupled (case 1, dashed line) and the locally coupled situation (case 2, solid line). The light dashed lines are linear fits (shifted in parallel for better visualization) obtained in the respective scaling regions.

case 1 , however, the two curves show different shapes. We attribute this to the fact that $D_{\mathrm{KLD}}$ is sensitive to both phase and amplitude turbulence, while the defect density captures the degree of disorder in the defect turbulent regime only. For example, when increasing $U$ from $U=1.06$ to $U=1.19$ the periodic limit cycle becomes chaotic due to irregular phase modulations [cf. Figs. 2(a) and 2(b)]. Hence, $D_{\mathrm{KLD}}$ exhibits an increase from 2 to 3 (cf. Fig. 4) while the defect density remains nearly constant (cf. Fig. 3).

A measure of the degree of spatial correlation is obtained from temporally averaged Fourier spectra of spatial profiles of the interfacial potential. Figure 5 shows two such power spectra in a log-lin diagram. The solid and dashed lines were obtained for fully developed turbulent time series of case 2 and case 1, respectively. Both curves are linear within a certain range of $k$, indicating a Lorentzian form of the autocorrelation function. The slopes of the linear fits are proportional to the full width at half maximum of the autocorrelation function and, therefore, can be regarded as a measure of spatial correlation in the data. Hence, the steeper slope in case 1 indicates a larger degree of spatial coherence, supporting our conclusion that in case 1 the length scale of dissipation or damping is larger than in case 2 as was inferred from Fig. 4. Since the dominant mechanism for damping is provided by the synchronizing effect of spatial coupling, we can deduce that the coupling range is larger in case 1 than in case 2 . This exactly matches the theoretical predictions for coupling in electrochemical systems: According to Eq. (1) the range of the spatial coupling depends on $\beta$, being nonlocal for a large distance between the two electrodes, and becoming more localized with decreasing distance. The results presented here give the first experimental support of this theoretically predicted peculiarity of spatial coupling in electrochemical systems.

In conclusion, we presented experimental data from an oscillatory electrochemical reaction that exhibited a tran- sition from uniform limit cycle oscillations via phase turbulence to defect turbulence. Thus, this transition scenario, formerly discussed extensively in the framework of the CGLE, also occurs in nonlocally coupled reactiontransport type systems far from a supercritical Hopf bifurcation, evidencing that this route to spatiotemporal chaos is more general than discussed so far. Moreover, the higher defect density and larger KLD dimension for a shorter distance between the working and the counterelectrode provide the first experimental proof that the spatial coupling range in electrochemical systems can indeed be tuned by varying the aspect ratio of the electrochemical cell.

We thank S. Mandelj for fruitful discussions. A. B. thanks the EU for support through the Marie Curie Fund.

*Electronic address: krischer@ph.tum.de

[1] M. C. Cross and P. C. Hohenberg, Rev. Mod. Phys. 65, 851 (1993).

[2] Y. Kuramoto, Chemical Oscillations, Waves and Turbulence (Dover Publication, Inc., Mineola, New York, 2003).

[3] I. S. Aranson and L. Kramer, Rev. Mod. Phys. 74, 99 (2002).

[4] B. I. Shraiman et al., Physica (Amsterdam) 57D, 241 (1992).

[5] E. Bodenschatz, W. Pesch, and G. Ahlers, Annu. Rev. Fluid Mech. 32, 709 (2000).

[6] Q. Ouyang and J.-M. Flesselles, Nature (London) 379, 143 (1996).

[7] Q. Ouyang and H. L. Swinney, Chaos 1, 411 (1991).

[8] J. Boissonade, E. Dulos, and P. DeKepper, in Chemical Waves and Patterns, edited by R.E. Kapral and K. Showalter (Kluwer, Dordrecht, 1995), p. 221.

[9] S. Jakubith et al., Phys. Rev. Lett. 65, 3013 (1990).

[10] G. Veser et al., Phys. Rev. Lett. 71, 935 (1993).

[11] M. Kim et al., Science 292, 1357 (2001).

[12] B. Marts et al., Phys. Rev. Lett. 93, 108305 (2004).

[13] K. Krischer, J. Electroanal. Chem. 501, 1 (2001).

[14] N. Mazouz, G. Flätgen, and K. Krischer, Phys. Rev. E 55, 2260 (1997).

[15] K. Krischer, in Advances in Electrochemical Sciences and Engineering, edited by D. M. Kolb and R.C. Alkire (Wiley-VCH, Weinheim, 2003), p. 89.

[16] J. Christoph et al., J. Chem. Phys. 110, 8614 (1999).

[17] F. Plenge et al., Z. Phys. Chem. 217, 365 (2003).

[18] G. Flätgen and K. Krischer, Phys. Rev. E 51, 3997 (1995).

[19] P.F. Panter, Modulation, Noise and Spectral Analysis (McGraw-Hill, New York, 1965).

[20] A. Pikovsky, M. Rosenblum, and J. Kurths, Synchronization (Cambridge University Press, Cambridge, 2001).

[21] M. Bertram et al., Phys. Rev. E 67, 036208 (2003).

[22] S. C. Zoldi and H. S. Greenside, Phys. Rev. Lett. 78, 1687 (1997).

[23] P. Grauel, H. Varela, and K. Krischer, Faraday Discuss. 120, 165 (2002). 